\title{
Publisher's Note: Overhead analysis of universal concatenated quantum codes [Phys. Rev. A 95, 022313 (2017)]
}

\author{
Christopher Chamberland, Tomas Jochym-O’Connor, and Raymond Laflamme \\ (Received 15 February 2017; published 24 February 2017)
}

DOI: 10.1103/PhysRevA.95.029904

This paper was published online on 8 February 2017 with an omission of an author affiliation and incorrect sizing of Fig. 5. Tomas Jochym-O'Connor's additional affiliation should read as "2Walter Burke Institute for Theoretical Physics and Institute for Quantum Information \& Matter, California Institute of Technology, Pasadena, California 91125, USA.” Figure 5 has been resized and the additional author affiliation has been added as of 15 February 2017. The figure is correct and the author affiliation is present in the printed version of the journal. 